\title{
Far fields, from electrodynamics to gravitation, and the dark matter problem
}

\author{
A. Carati $^{1}$, S.L. Cacciatori ${ }^{2}$, and L. Galgani ${ }^{1}$ \\ 1 Department of Mathematics, Milan University, Via Saldini 50, I-20133 Milano, \\ Italy. andrea.carati@unimi.it, luigi.galgani@unimi.it \\ 2 Department of Physical and Mathematical Sciences, Insubria University, Via \\ Valleggio 11, I-22100 Como, Italy. sergio.cacciatori@uninsubria.it
}

Summary. We describe the steps we followed in obtaining a recent result, according to which it may not be necessary to make recourse to dark matter in order to explain the observed velocity dispersion in the Coma cluster. The main idea is that the missing gravitational action may be due to the external far galaxies. In particular, we describe how relevant for us was the analogy between the retarded far fields of electrodynamics and those of gravitation theory, in order to understand the dominant role of the far galaxies.

\section{Introduction}

Very recently it occurred to us to write a paper (see [1]) in which it was pointed out that the existence of a dark matter may not be necessary in order to explain the phenomenology, at least in the application of the virial theorem to the Coma cluster, which is the first case where the existence of a dark matter was conceived (by Zwicky, in the year 1933; see [2]). The point we made is that one should take into account the gravitational force due to the external galaxies, which usually are not even mentioned at all. We gave an estimate for such a force, showing that it seems to be of the right order of magnitude. Three points play a fundamental role in our argumentation. Two of them (Hubble's law and the fractal nature of the Universe) are of a cosmological character. Preliminarily, there is however a point of a kinematic-dynamic character. This concerns the role played in the theory of gravitation by the far fields, inasmuch as they are the ones that give the dominant contribution to the force on a test particle (especially if Hubble's law is taken into account). Now, it is true that the far fields are a standard topic in the theory of gravitation, in connection with the problem of the gravitational waves. However, the idea that they may be relevant for the dynamics of the galaxies seems to be new. In the present paper we describe first how it occurred to us to come to such a conception by analogy with the case of electrodynamics, and then how we finally came to our result. 
The recognition of the relevance of the analogy between far fields in electrodynamics and in gravitation actually had a rather occasional origin. During a conference in honour of Claude Froeschlé, held in Spoleto at the end of June 2007 , one of us had the opportunity to illustrate some recent results concerning the classical microscopic theory of matter-radiation interaction for a system of dipoles located at the sites of an infinite lattice [3] [4]. Particular emphasis was given to two features, namely, the retarded character of the forces and the global character of the interactions of the infinitely many dipoles composing the system. Such features had indeed played an essential role in the deduction of the main result, i.e., a proof of the Wheeler-Feynman identity. It then naturally occurred that, in a meeting mostly devoted to celestial mechanics, the question would be raised whether such features concerning retardation, the occurring of far fields and the global character of the interactions may perhaps play some role also in the theory of gravitation. Many discussions in this connection took place with George Contopoulos and Christos Efthymiopoulos, and these were particularly stimulating for us.

In the meantime, it had occurred to us to read, in the occasion of the thesis of a student (see [5]) the papers in which Zwicky was applying the virial theorem to the Coma cluster, while at the same time we were lecturing on the classical application of such a theorem to gases by Clausius. The comparison between the two applications then came up very naturally, with the realization of the lack of any justification for the neglect of the external forces in the case of Zwicky. So the circle was closed, and the idea was formed that one should take into account the gravitational force exerted by the external galaxies. In particular, one had to understand how it may happen that such a force can have the character of a pressure, thus playing a role analogous to that of the walls in the case of gases. Here, it will be described how, step by step, we came to our final estimate.

\section{Far fields in electrodynamics, and the Wheeler Feynman identity}

The recognition of the global role of the far fields in electrodynamics came about as follows. In the paper [6] it was pointed out that there is a deep inconsistency in the way Planck was dealing, in his classical papers, with the dynamical aspects of the black-body problem. Indeed, he was studying the interaction of a "material resonator" with the electromagnetic field (which in some approximation could be reduced to just the electric field E). He had previously understood (possibly being the first one to do so) that the "self-interaction" of the material resonator with the field should be taken into account through an effective self-force proportional to the third derivative of its position. However, for the sake of simplicity Planck introduced the approximation in which he replaced such a self-force with a damping force proportional to the velocity. So for the resonator he wrote down an equation 
of the type

$$
m\left(\ddot{\mathbf{q}}+\omega_{0}^{2} \mathbf{q}+\omega_{0}^{2} \tau \dot{\mathbf{q}}\right)=\mathbf{E}(t)
$$

where $\mathbf{q}$ is the position vector of the resonator and $\mathbf{E}(t)$ the external electric field acting on it; moreover, $\omega_{0}$ a characteristic angular frequency of the resonator, while $\tau=(2 / 3) e^{2} /\left(m c^{3}\right)$ is the familiar characteristic time of the classical electron theory, $c$ being the speed of light and $e$ and $m$ the charge and the mass of the resonator. An equation of such a type is discussed by Planck for example in the celebrated paper where his "second theory" was first advanced. One can check how Planck introduced some generic assumptions for the function $\mathbf{E}(t)$, without taking into account the fact that such a field is the one produced by the other resonators composing the considered system. This should enforce one to deal with a many-body problem, whereas Planck was explicitly assuming (see the quotation in [6], and [7]) that all the resonators were acting "independently of one another".

The mutual retarded interactions among all resonators was taken into account in the paper [3], where an extremely simple many-body model was introduced. This is a system of infinitely many dipoles each of which can oscillate about a site of an infinite regular chain (and along the direction of the chain), being attracted to its equilibrium position by a linear restoring force. Moreover, each dipole is subject to a retarded interaction with all the other ones through an electric force which is a solution of the Maxwell equations having all the other dipoles as sources. The system is then linearized, inasmuch as the distance between the dipoles is approximated by the distance between the corresponding equilibrium points. Moreover, for what concerns the selfforce, no approximation is made, and its standard form, proportional to the third derivative, is used. If $q_{j}$ denotes the displacement of the $j$-th dipole from its equilibrium position, then one has the infinite system of equations with delay given by

$$
m\left(\ddot{q}_{j}+\omega_{0}^{2} q_{j}-\tau \dddot{q}_{j}\right)=2 e^{2} \sum_{k \neq j}\left[\frac{q_{k}\left(t-r_{j k} / c\right)}{r_{j k}^{3}}+\frac{1}{c} \frac{\dot{q}_{k}\left(t-r_{j k} / c\right)}{r_{j k}^{2}}\right],
$$

where $r_{j k}=a|j-k|$ is the distance between the equilibrium positions of dipoles $j$ and $k, a$ being the lattice step.

In the paper [3] the rather astonishing result was proven that there exist normal-mode solutions of the complete system, namely, solutions of the type $q_{j}(t)=A \exp (\kappa a j-\omega t)$ with a suitable dispersion relation $\omega=\omega(\kappa)$. Thus, in such a solution there occurs for each dipole an exact compensation between the energy it emits and the energy it receives from all the other ones. Such an exact compensation occurs in virtue of an identity which had already been conceived by Oseen [8] in the year 1916, and is essentially equivalent to the more familiar one that was amply discussed by Wheeler and Feynman [9] in the year 1945. An essential point concerning such an identity is its global character, inasmuch as it makes reference to the whole system of all dipoles. 
This feature becomes even more evident if one considers the analogous three-dimensional model (see [4]), because in such a case the main contribution to the force acting on a single dipole is the one coming from the far ones. This is due to the fact that the four-potential acting on a dipole is the retarded solution of the wave equation having each of the other dipoles as a source. Thus, concerning the force "created" by any other dipole, in addition to the Coulomb term there appear both the "near" term proportional to the velocity of the source, which decays as $1 / r^{2}$, and the "far" one proportional to the acceleration of the source (and normal to its velocity), which decays as $1 / r$. The latter term, which is the dominating one, was actually lacking in the one-dimensional model, due to the accidental fact the dipoles were constrained to oscillate along the direction of the chain. So we have described how we came to understand the role of far fields in electrodynamics.

\section{The virial theorem in the theory of gases and in astrophysics, and the role of the external forces}

Let us now come to the problem of gravitation, and start up recalling how the virial theorem was applied by Zwicky to the Coma cluster, in contrast to the classical application to gases made by Clausius. One considers a system $S$ composed of $n$ points. For Clausius $S$ is a gas enclosed in a box, for Zwicky it is the Coma cluster, whose "points" are galaxies, immersed in the Universe. One considers Newton's equations of motion $\ddot{\mathbf{x}}_{i}=\mathbf{F}_{i} / m_{i} \equiv \mathbf{f}_{i}, i=1, \ldots, n$ (the dot denoting time derivative) where $\mathbf{x}_{i}$ is the position vector of the $i$-th particle with respect to the center of mass of the system $S$, while $\mathbf{F}_{i}$ is the force acting on the $i$-th particle (of mass $m_{i}$ ) and $\mathbf{f}_{i}=\mathbf{F}_{i} / m_{i}$ is the corresponding force per unit mass, i.e., the corresponding acceleration. Then one takes the dot product with $\mathbf{x}_{i}$, and adds over $i$. Performing a time average, under the hypothesis that the system remains confined one immediately obtains

$$
\overline{\sigma_{v}^{2}}=-\overline{\mathcal{V}} / n
$$

where $\sigma_{v}^{2}=(1 / n) \sum_{i} v_{i}^{2}$ is the variance of the velocity distribution of the galaxies of the cluster, whereas $\mathcal{V}=\sum_{i} \mathbf{f}_{i} \cdot \mathbf{x}_{i}$ is called the virial of the forces (per unit mass), and overline denotes time-average. This is the form of the theorem suited to the case of gravitation (which involves forces per unit mass), whereas the analogous theorem for the case of general forces relates twice the kinetic energy to the virial of the forces.

Notice that one has the decomposition $\mathbf{F}_{i}=\mathbf{F}_{i}^{i n t}+\mathbf{F}_{i}^{e x t}$ of the force acting on the $i$-th particle as the sum of an internal force and an external one (that exerted by the walls of the box confining the gas in the case of Clausius, and by the galaxies external to Coma in the case of Zwicky). In the case of Clausius one has $\mathbf{F}_{i}^{i n t}=0$ for a perfect gas, and in any case the virial of the internal forces is considered to be negligible with respect to the external 
one. In turn, the virial of the external forces is related to the pressure, and so from the virial theorem one obtains the thermodynamic interpretation of the translational kinetic energy as proportional to temperature. In the case of Coma, instead, Zwicky doesn't make any mention of the external forces at all, and considers only the internal ones, given by Newton's gravitational law. He thus finds, analogously to the case of gases, that $\left|\overline{\mathcal{V}^{i n t}}\right| / n$ is just a negligible fraction of $\sigma_{v}^{2}$, and so he is led to the conjecture that some non visible mass exists, whose contribution to the virial may restore the balance with the observed velocity variance.

Now, why should the external forces be neglected at all? The first idea underlying our work was that in astrophysics, just as in the case of gases, the virial of the external forces may actually be what is needed in order to restore the balance in the virial theorem.

\section{Relevance of the far matter if Hubble's law is taken into account}

So we had the problem of estimating the gravitational field of force due to the external galaxies. From the point of view of general relativity, in the weak-field approximation this amounts to writing down the equations for the geodesic motion when the metric tensor $g_{\mu \nu}$ is a solution of the Einstein equation with the external galaxies as sources. It is well known that, for small velocities of the test particle, the equation for the geodesic motion is the same as for a point particle with a Lagrangian

$$
L=g_{i k} \dot{x}^{i} \dot{x}^{k}+c g_{0 k} \dot{x}^{k}+c^{2} g_{00},
$$

where $c$ is the speed of light (the summations over the spatial indices $i$ and $k$ from 1 to 3 being understood). So at first sight, neglecting the corrections due to the kinetic energy, the forces per unit mass appear to be the same as if the test particle were in presence of an electromagnetic field having $g_{00}$ as scalar potential and $g_{0 k}$ as vector potential (although relevant differences exist between the two cases, as particularly emphasized by Zeldovich and Novikov [10]). On the other hand, it is very well known that in the weak-field approximation, writing the metric tensor as a perturbation of the Lorentzian background $\eta_{\mu \nu}$, namely, as $g_{\mu \nu}=\eta_{\mu \nu}+h_{\mu \nu}$, the perturbation $h_{\mu \nu}$ turns out to be a solution of the wave equation, so that its components are the familiar retarded potentials of electrodynamics. In fact one finds

$$
h_{\mu \nu}=\left.\frac{-2 G}{c^{4}} \sum_{j=1}^{N} M_{j} \frac{1}{\gamma_{j}} \frac{2 \dot{q}_{\mu}^{(j)} \dot{q}_{\nu}^{(j)}-c^{2} \eta_{\mu \nu}}{\left|\mathbf{x}-\mathbf{q}^{(j)}\right|}\right|_{t=t_{r e t}},
$$

where $G$ is the gravitational constant, while $M_{j}, \mathbf{q}^{(j)}$ and $\gamma_{j}, j=1, \ldots, N$, are the mass, the position vector and the Lorentz factor of the $j$-th source 
galaxy, dealt with as a point particle, and the dot denotes derivative with respect to proper time along the worldline of the source. The notation $\mathbf{q}^{(j)}$ in place of $\mathbf{q}_{j}$ for the position vector of the $j$-th external galaxy was introduced just in this formula, in order to avoid confusion with the tensorial indices.

Formula (4) implies first of all that, according to general relativity in the weak-field approximation, the gravitational field of force presents (in analogy with electrodynamics) both a "classical" Newtonian near-field term, decaying as $1 / r^{2}$, and a far-field one, decaying as $1 / r$. This in turn implies that the contribution to the gravitational field of force due to the far matter is in principle dominant with respect to that due to the near matter. In other terms, the problem of estimating the external gravitational field of force acting on a localized system such as the Coma cluster, immediately presents itself as a problem of a cosmological character, and this compels one to introduce a cosmological model.

To this end we introduced an oversimplified model. Let us make reference to a local chart with Lorentzian coordinates, having as origin the center of mass of the considered localized system (the Coma cluster). As $h_{\mu \nu}$ depends on each source not only through its position $\mathbf{q}_{j}$, but also through its velocity, the latter has to be assigned in order that the model be defined. In our simple model, this is obtained by introducing Hubble's law as a phenomenological prescription, namely, by requiring that for the external galaxies one has

$$
\dot{\mathbf{q}}_{j}=\gamma_{j}^{-1} H_{0} \mathbf{q}_{j}, \quad j=1, \ldots, N
$$

(the dot denoting now derivative with respect to the background Lorentzian time). Here, $H_{0}$ is the Hubble constant which, in our extremely simplified model, we take fixed to its present value.

Notice that the Hubble assumption (5) has an essential impact on the size of the gravitational field of force. Indeed, in the field of force one has a term (decreasing as $1 / r^{2}$ ) proportional to the velocity of the source, and a term (decreasing as $1 / r$ ) proportional to the acceleration of the source. Thus, as Hubble's law (5), implies that also the acceleration has a contribution proportional to the distance, it turns out that the term proportional to the acceleration actually doesn't depend on distance at all. This is the main reason why the far matter gives the dominant contribution to the gravitational field of force.

The force per unit mass at the origin corresponding to such a dominant term (which we denote by $\mathbf{f}$ ) turns out to have the form

$$
\mathbf{f}=\frac{4 G H_{0}^{2} M}{c^{2}} \mathbf{u}
$$

where we have introduced the vector $\mathbf{u}$, depending on the number $N$ of external galaxies, defined by

$$
\mathbf{u}(N)=\sum_{j=1}^{N} \frac{\mathbf{q}_{j}}{\left|\mathbf{q}_{j}\right|} .
$$


Here, the masses of the sources were all put equal to a common value $M$, and the Lorentz factors $\gamma_{j}$ were approximated to 1 . As will be shown later, this approximation is justified for the aims of our estimate. Notice the extremely simple nature of this force per unit mass: apart from a multiplicative factor, it is just the sum of all the unit vectors pointing to each of the $N$ external galaxies.

Concerning the main procedure which was followed in obtaining the force exerted by the external matter, the following comment may be in order. The idea of taking into account the role of the external matter was discussed by Einstein, in his Princeton lectures of the year 1921 (see [11]), in connection with the Mach principle, where he pointed out that the perturbation $h_{\mu \nu}$ to the metric (that he was denoting by $\gamma_{\mu \nu}$ ) can be obtained "by the method. familiar in electrodynamics, of retarded potentials " (see his formula (101) at page 87). The only difference is that at those times he did non yet had available Hubble's law, and on the other hand he had in mind the application to astronomy. So he wrote (page 88) "The previous developments are valid however rapidly the masses which generate the field may move relatively to our chosen system of quasi-Galilean coordinates. But in astronomy we have to do with masses whose velocities, relatively to the coordinate system employed, are always small compared to the velocity of light,.... We therefore get an approximation which is sufficient for nearly all practical purposes if in (101) we replace the retarded potential by the ordinary (non-retarded) potential ...". This is the way it happened that only the Newtonian, fast decaying, potential was considered, and consequently only the near matter, and not the far one, did play a role in connection with Mach's principle (see page 100 of ref. [11]).

\section{Estimate of the gravitational forces. Role of the discreteness of the sources, and of the fractal nature of the Universe}

So we had to estimate the gravitational force per unit mass exerted by the external galaxies, namely essentially the sum (7), and we now briefly describe the steps we followed. The first result was a negative one: One shows that the external force exactly vanishes if the external matter is described as a continuum, with a radially symmetric density.

As a further step, we took into account the fact that the matter actually is a discrete system of point particles. In order to make an estimate with concrete sets of positions for the galaxies, we took the probabilistic point of view that Chandrasekhar and von Neumann (see the review [12]) had taken in order to estimate the vector sum of the Newtonian forces exerted on a star by the near ones. Following such authors, we started assuming that the positions $\mathbf{q}_{j}$ of the $N$ galaxies are independent random variables, uniformly distributed with respect to the Lebesgue measure. Then the sum (7) is found to grow as $\sqrt{N}$, just as a consequence of the central limit theorem. Correspondingly, apart 
from a constant factor, the force per unit mass (6) behaves, with increasing $N$, as the fraction $M N / \sqrt{N}$, where the total mass $M N$ of the galaxies was put in evidence in the numerator. One easily checks (see later) that such a force is completely negligible.

So we modified the previous assumption, and considered the case in which the distribution of mass is assumed to be fractal [13] (see also [14], [15], [16]. [17]). This means first of all that the positions of the galaxies are no more independently distributed, and this has the relevant consequence that the sum (7) is no more constrained to grow as $\sqrt{N}$, and can instead have a faster growth, as required by the observations. The removal of the assumption of independence of the positions of the galaxies has however the consequence that the analytical discussion becomes now much more difficult than in the case of Chandrasekhar and von Neumann. So we were forced, at least provisionally, to investigate the problem by numerical methods.

We proceeded as follows. We actually considered the component of the force $\mathbf{f}$ (or of the corresponding vector $\mathbf{u}$ ) along a given direction. Such a component of $\mathbf{f}$ was simply denoted by $f$ and, analogously, the corresponding component of $\mathbf{u}$ by $u$. To estimate the sum, the positions of the galaxies were extracted (with the method described in [13]) in such a way that the mass distribution has a fractal dimension, precisely the fractal dimension 2 . The quantity $u$ was thus dealt with as a random variable, and its probability distribution was investigated by considering 10,000 samples, with $N$ ranging from 1000 to 512,000 , the density being kept constant. This means that the positions of the $N$ points were taken to lie inside a cutoff sphere whose volume was made to increase as $N$. For the values of $N$ investigated, the corresponding radius turns out to be so small with respect to the present horizon, that the Lorentz factors $\gamma$ could altogether be put equal to 1 .

The mean of $u$ was found to practically vanish for all $N$, while its variance $\sigma_{u}^{2}$ was found to grow as $N^{2}$ (actually, as $0.2 N^{2}$ ), rather than as $N$, as occurs in the uniform case (see Fig. (2) of ref [1]). We thus could conclude that the standard deviation $\sigma_{f}$ of the component of the force per unit mass along a direction is proportional to $N$, being given by

$$
\sigma_{f} \simeq \sqrt{0.2} \frac{4 G H_{0}^{2}}{c^{2}} M N=\sqrt{0.2} \frac{4 G}{R_{0}^{2}} M N,
$$

where $R_{0}=c / H_{0}$ is the present horizon. We then took such a result, which was obtained for extremely small values of $N$, and extrapolated it up to the present horizon $R_{0}=c / H_{0}$, i.e., we inserted in formula (8) the actual value of $N$, so that the quantity $M N$ could be identified with the total visible mass of the Universe.

Concerning the total visible mass $M N$ of the Universe, one can write

$$
M N=\frac{4}{3} \pi \rho_{\mathrm{eff}} R_{0}^{3},
$$


with a suitable effective density $\rho_{\text {eff }}$. In the paper [1] it was shown that a natural consistency condition for the model gives

$$
\rho_{\text {eff }}=\frac{1}{4} \frac{3 H_{0}^{2}}{8 \pi G} \simeq 5 \rho_{0}
$$

where $\rho_{0}=\Omega_{0}\left(3 H_{0}^{2}\right) /(8 \pi G)$, with $\Omega_{0} \simeq 0.05$, is the actual density. This gives $\sigma_{f} \simeq 0.2 \mathrm{cH}_{0}$. On the other hand, if a random variable $f$ has zero mean and a finite variance $\sigma_{f}^{2}$, with great probability it will take on values very near to its standard deviation $\sigma_{f}$. In such a sense we may say we have found for the force per unit mass along a direction, exerted by the external galaxies, the typical value

$$
|f| \simeq 0.2 c H_{0} .
$$

\section{Application to the virial for a cluster of galaxies}

We finally applied such a result to estimating the virial for a cluster of galaxies. This could be done at a very heuristic level. First of all, one has to assume that locally, in the region of interest, the field of force has somehow a central character, because otherwise the cluster itself could not exist at the considered place. In other words, we are assuming that, for a given realization of the positions of the external galaxies, on the average (over the positions of the internal galaxies) locally the field of force is directed towards a center, i.e., is acting as a pressure. However, apart from such a correlation, the intensity of the field of force should not be thought of as a smooth function, being for the rest uncorrelated with respect to the position.

Let us consider a cluster composed of $n$ galaxies. We have to estimate the time-average of the virial of the forces (per unit mass) due to the $N$ external galaxies, namely, the quantity

$$
\overline{\mathcal{V}}=\sum_{i=1}^{n} \overline{\mathbf{f}_{i} \cdot \mathbf{x}_{i}},
$$

where $\mathbf{f}_{i}$ is the force on the $i$-th internal galaxy due to the external ones. We can assume all terms of (10) to be equal, and given by $\overline{\mathbf{f}_{i} \cdot \mathbf{x}_{i}} \simeq-f \overline{\left|\mathbf{x}_{i}\right|}$ with $f$ given by (9), while taking $\overline{\left|\mathbf{x}_{i}\right|} \simeq L / 4$, where $L$ is the diameter of the cluster. So one finds $|\overline{\mathcal{V}}| \simeq n f L / 4$. Inserting for $f$ the expression (9), one thus obtains the result that, according to the virial theorem, if the external force due to the far galaxies is taken into account, the velocity variance of a cluster should obey the law

$$
\overline{\sigma_{v}^{2}} \simeq 0.2 \frac{c H_{0} L}{4},
$$

where $L$ is the linear dimension of the cluster. In the case of Coma, for the velocity variance one thus finds a value $\simeq 6 \cdot 10^{5} \mathrm{~km}^{2} / \mathrm{sec}^{2}$, which is very near to the value $5 \cdot 10^{5} \mathrm{~km}^{2} / \mathrm{sec}^{2}$ reported by Zwicky. 
Notice the linear dependence on $L$ in formula (11), which seems to be in agreement with the observations (see [18], Fig. 2, page 539). This property is also confirmed by a dimensional analysis. Indeed, with the parameters entering the problem, the square of a velocity can be formed only as $c^{2}$, or as $c H_{0} L$ or as $\left(H_{0} L\right)^{2}$. But the first term is by far too large, the last term by far too small, while the term linear in $L$ is indeed about of the correct order of magnitude.

\section{Conclusion}

So we have described how, starting from the analogy with electrodynamics, we were led to conceive that the gravitational action of the far galaxies may be a substitute for the dark matter, if Hubble's law is taken into account. We also emphasized the role that the fractal nature of the Universe has in allowing for the corresponding force per unit mass to have the right order of magnitude, namely, about $\mathrm{cH}_{0}$.

This paper is dedicated to the memory of Nikos Voglis

\section{References}

1. Carati, A., Cacciatori, S.L., Galgani, L.: Far fields and dark matter, arXiv:0712.0547v3[astro-ph].

2. Zwicky, F.: Helv. Phys. Acta 5, 110 (1933); A. Phys. J. 86, 217 (1937).

3. Carati, A., Galgani, L.: Nuovo Cim. B 118, 839 (2003), arXiv:physics /0312075v1[physics.optics].

4. Marino, M, Carati, A., Galgani, L.: Annals of Physics 322, 799 (2007).

5. Giaz, A.: Thesis in Physics, University of Milan (2007).

6. Carati, A., Galgani, L.: Int. Journ. of Mod. Phys. B 18, 549 (2004).

7. Planck, M.: The theory of heat radiation (Dover, New York 1959).

8. Oseen, C.W.: Physik. Zeitschr. 17, 341 (1916).

9. Wheeler, J.A., Feynman, R.: Rev. Mod. Phys. 17, 157 (1945).

10. Zeldovich, Ya.B., Novikov, I.D.: Stars and relativity (Dover, New York 1971).

11. Einstein, A.: The meaning of relativity (Princeton U.P., Princeton 1922).

12. Chandrasekhar S.: Rev. Mod. Phys. 15, 1 (1943).

13. Mandelbrot, B.: The fractal geometry of nature (Freeman, New York 1977).

14. Pietronero, L.: Physica A 144, 257 (1987).

15. Ruffini, R., Song, D.J., Taraglio, S.: Astron. Astrophys. 190, 1 (1988).

16. Sylos-Labini, F., Montuori, M., Pietronero, L.: Phys. Rep. 293, 61 (1998).

17. Combes, F.: Astrophysical fractals: interstellar medium and galaxies. In The chaotic Universe. ed. by Gurzadyan, V.G., Ruffini, R. (World Scientific, Singapore 2000).

18. Kazanas, D., Mannhein, P.D.: Dark matter or new physics ? In After the first three minutes, AIP Conference Proceedings 222, ed. by Holt, S., Bennett, C.L., Trimble, B.V. (American Institute of Physics, New York 1991). 